\title{
Short-term and long-term outcomes of liver resection for HCC patients with portal vein tumor thrombus
}

\author{
Lei Huo ${ }^{1,2 \dagger}$, Wenxin Wei ${ }^{1 \dagger}$, Zhenlin Yan ${ }^{1 \dagger}$, Zhengqing Lei ${ }^{1,3}$, Yanting Xie ${ }^{1}$, Renyan Gong ${ }^{1}$, Shengyu Huang ${ }^{1}$,
} Ningyang Jia ${ }^{2^{*}}$ and Yong $\mathrm{Xia}^{1 *}$

\begin{abstract}
Background: Portal vein tumor thrombosis (PVTT) in hepatocellular carcinoma (HCC) is a sign of advanced stage disease, which is associated with poor prognosis. Liver resection (LR) may provide better prognosis in selected patients. In the present study, we aimed to assess information from HCC patients with PVTT who died within 3 months or 2 years after $L R$ in order to identify preoperative factors correlated to short-term or long-term survival, by which inappropriate selection of patients for LR might be avoided in the future.

Methods: A retrospective cohort study consisting of 487 consecutive cases of HCC patients with PVTT was performed from 2008 to 2010 at Eastern Hepatobiliary Surgery Hospital. Medical records, including laboratory values, imaging results and treatment information, were obtained from participants. Study endpoints were survival at 3 months and 2 years post-hepatectomy. Logistic regression analysis was utilized to determine the significant preoperative factors influencing short-term or long-term survival.

Results: In multivariable analysis, a-fetoprotein, total bilirubin and radiologic ascites were significantly associated with short-term survival, while a-fetoprotein level, clinical significant portal hypertension, extent of PVTT and tumor differentiation were factors significantly associated with long-term survival.

Conclusions: The independent risk factors of poor short-term survival were the liver function-associated, such as factors radiologic ascites and total bilirubin, while tumor differentiation indicating the tumor biology was associated with longer-term survival. In addition, a-fetoprotein was a risk factor associated with both short-term and longer-term survivals.
\end{abstract}

Keywords: Hepatocellular carcinoma, Portal vein tumor thrombosis, Hepatectomy, Prognosis

\section{Background}

Previous study has shown that patients with hepatocellular carcinoma (HCC) and portal vein tumor thrombus (PVTT) have an extremely poor prognosis [1]. A huge proportion of HCC patients (12.5-39.7\%) exhibit gross

\footnotetext{
*Correspondence: ningyangjia@163.com; xia.yong@126.com

'Lei Huo, Wenxin Wei and Zhenlin Yan contributed equally to this work

${ }^{1}$ Department of Hepatic Surgery IV, The Eastern Hepatobiliary Surgery Hospital, Second Military Medical University, 225 Changhai Road,

Shanghai 200433, China

${ }^{2}$ Department of Radiotherapy, The Eastern Hepatobiliary Surgery

Hospital, Second Military Medical University, Shanghai 200438, China

Full list of author information is available at the end of the article
}

PVTT at the time of diagnosis, while such occurrence of PVTT becomes $44 \%$ at the time of death [2]. In addition, the median survival is $2.7-4.0$ months when tumor remains untreated [3].

The optimal treatment for HCC patients with PVTT remains controversial. As a standard therapy, sorafenib has been widely used for advanced HCC with PVTT or metastasis. In Asian countries, the median survival is 6.5 months when patients are administered with sorafenib [4]. Many clinical guidelines have shown that hepatectomy is a safe and effective treatment for HCC patients with PVTT if the patients are carefully selected [5-7]. Surgical treatment may offer a chance of cure, 
and post-operative 5-year overall survival (OS) has been reported to range from 10 to $59 \%$ [8-10]. A median survival ranging from 8 to 22 months has been reported for HCC patients with PVTT after surgical treatment [11, 12].

Recently, more aggressive therapeutic schemes, such as operation, interventional radiotherapy, molecularly targeted therapy and other multidisciplinary treatments, have been developed, leading to improved life quality and a prolonged survival time in some patients. As a result of recent advances in surgical techniques and peri-operative management, liver resection (LR) has become a reasonably safe treatment option with an acceptable mortality and morbidity rate $[8,9]$. Several tertiary centers have proposed aggressive surgical resection for $\mathrm{HCC}$ with vascular invasion [13].

Now, hepatic surgeons may perform palliative surgery in order to improve quality of life, by which the patients can be reasonably to survive for at least 3 months. When patients have a life expectancy $<3$ months, such population is generally unsuitable for surgery because the risks and drawbacks, including pre- and post-operative complications, management of post-operative pain, and time required for recovery, usually offset the benefits of intervention, and patients are subsequently referred for systemic therapy or palliative medical care.

In order to recognize which patient will not benefit from surgery, it would be valuable to identify pre-operative factors associated with exceedingly short survival.

In the present study, we aimed to compare clinical presentation and outcomes in patients with $\mathrm{HBV}$-associated HCC, stratified by the presence or absence of cirrhosis and symptoms at time of diagnosis. Patients who died within 3 months of surgery were compared with those long-term survivors ( $>2$ years). If the patients died within 3 months of surgery, they were inappropriate for surgery. Therefore, we attempted to identify pre-operative factors, which might indicate that anticipated survival does not justify the risk of surgery.

\section{Results}

\section{Baseline clinicopathological characteristics}

A total of 457 patients were included in the database. The cohort used in the analysis was under ongoing follow-up. Of the 457 patients, 456 patients were included in the 3-month survival analysis, and 389 patients were included in the 2-year survival analysis (Fig. 1). Univariable and multivariable regression analyses were exclusively conducted on these two smaller groups. The average age of the patients at time of surgery was $47.9 \pm 10.3$ years, and males accounted for $89.1 \%$ of the study population. Table 1 lists detailed baseline characteristics of all patients.

\section{Patients who survived $<3$ months}

Descriptive analysis showed that patients who died within 3 months were significantly associated with a larger diameter, higher bilirubin and higher AFP level. Table 2 displays detailed descriptive analysis of the two groups of patients at 3 months post-hepatectomy.

Patients who had a follow-up visit at, at least, within 3 months or were discharged at this point $(n=405)$ were compared with those who died within 3 months $(\mathrm{n}=51)$, and results showed the factors associated with death within 3 months in univariable analysis (Additional file 1: Table S1). In multivariable analysis, AFP, per Log $10 \mu \mathrm{g} / \mathrm{L}$ increase (odds ratio [OR], 1.60; 95\% CI 1.15 to $2.25 ; \mathrm{P}=0.010)$, TBIL, per $\mathrm{mg} / \mathrm{dL}$ increase $(\mathrm{OR}, 1.94 ; 95 \%$ CI 1.21 to $3.12 ; \mathrm{P}=0.026)$ and positive radiologic ascites (OR, 3.72; 95\% CI 1.59 to $8.15 ; \mathrm{P}=0.009$ ) were the significant factors associated with death within 3 months (Table 3). A total of 51 patients died within 3 months because of systemic progression of cancer $(n=146$; $84.4 \%$ ), complications of spine surgery within 30 days of surgery $(n=7 ; 4.0 \%)$, and unknown or unspecified reasons $(n=20 ; 11.6 \%)$.

\section{Patients who survived $>\mathbf{2}$ years}

Descriptive comparison between patients who survived $>2$ years $(\mathrm{n}=98)$ post-hepatectomy and those who died within 2 years $(n=291)$ after surgery revealed that survivors had a favorable pre-operative condition characterized by lower AFP, better pre-operative liver function, smaller tumor diameter and more favorable tumor differentiation (Table 4).

Patients who survived for at least 2 years $(n=98)$ were compared with those who died within 2 years after surgery $(n=291)$, and results showed that the following factors were associated with death within 2 years after surgery (Additional file 1: Table S1). In multivariable analysis, AFP, per Log $10 \mu \mathrm{g} / \mathrm{L}$ increase (OR, 1.34; 95\% CI 1.06 to $1.69 ; \mathrm{P}=0.034)$, clinically significant portal hypertension $(\mathrm{CSPH})(\mathrm{OR}, 1.75 ; 95 \% \mathrm{CI} 1.10$ to $2.81 ; \mathrm{P}=0.034)$, extent of PVTT (OR, 1.81; 95\% CI 0.99 to 3.53 ; $\mathrm{P}=0.043$ ) and tumor differentiation III-IV (OR, 3.93; 95\% CI 1.56 to $10.18 ; \mathrm{P}=0.017)$ were significantly associated with death within 2 years.

\section{Effects of AFP and TBIL on survival in cirrhosis context}

Survival was compared across subgroup based on patients' cirrhosis context. In the subset of patients with cirrhosis, the 1-, 3- and 5-year OS rate for patients with low or high serum AFP level was $47.9 \%, 22 \%$ and $18 \%$ or $34.2 \%, 16.3 \%$ and $10.2 \%$, respectively $(\mathrm{P}=0.0398$, Fig. $2 \mathrm{a})$, while for patients with low or high serum TBIL level it became $39.3 \%, 17.9 \%$ and $13.2 \%$ or $38.2 \%, 19.1 \%$ and $13.6 \%$, respectively ( $\mathrm{P}=0.242$, Fig. $2 \mathrm{~b}$ ). In the subset of 
Hepatic vein tumor thrombus $(\mathrm{n}=51)$

Bile duct tumor thrombus $(\mathrm{n}=20)$

Inferior vena cava tumor thrombus $(\mathrm{n}=2)$

Abdominal metastasis $(n=15)$

Missing data regarding their survival time $(\mathrm{n}=10)$

Pathology confirmed HCC

patients with PVTT $(\mathrm{n}=555)$
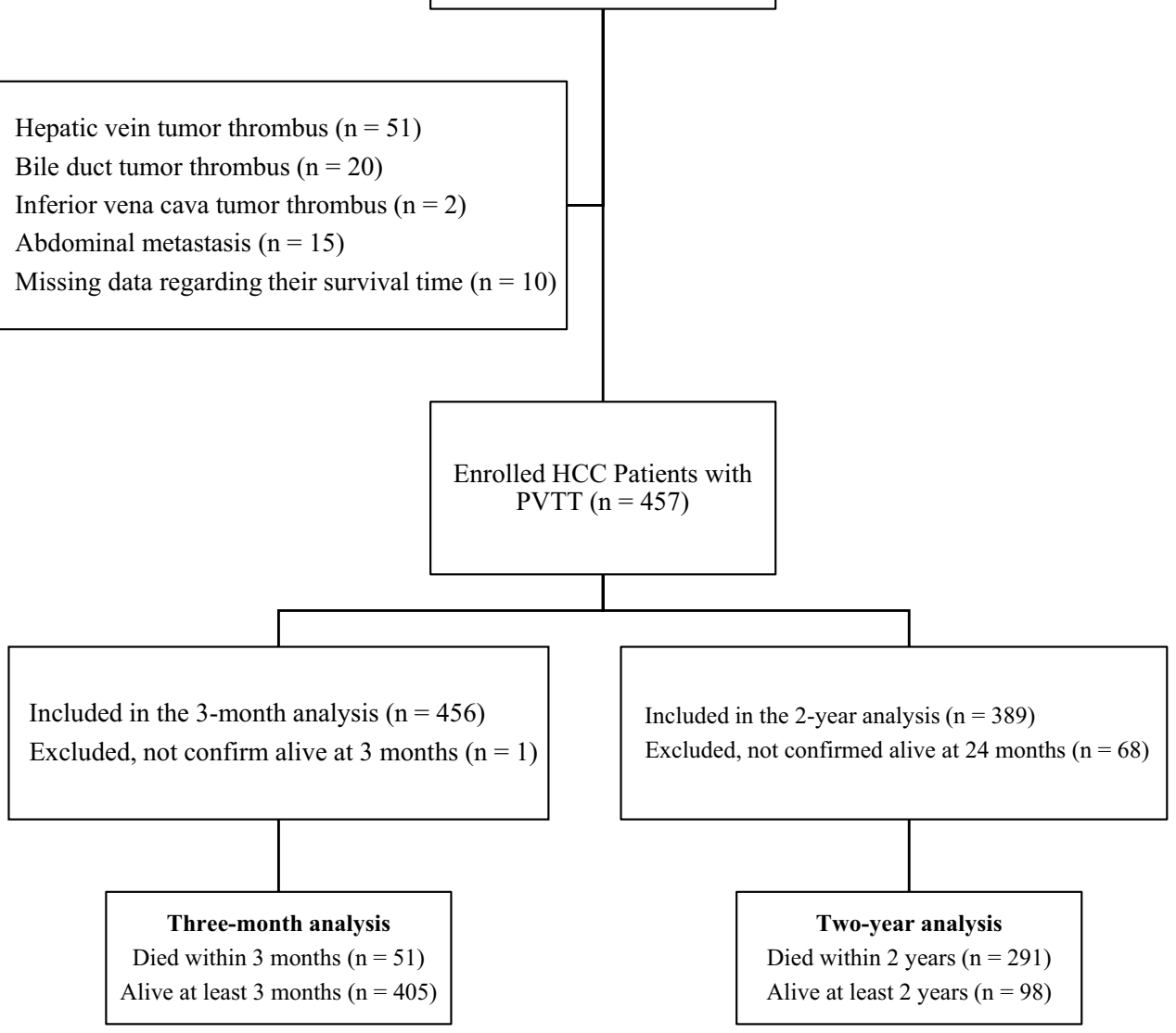

Fig. 1 Flow diagram

patients without cirrhosis, the 1-, 3- and 5-year OS rate for patients with low or high serum AFP level was 57.9\%, $32.6 \%$ and $22 \%$ or $35.20 \%, 19.4 \%$ and $16.1 \%$, respectively $(\mathrm{P}=0.049$, Fig. $2 \mathrm{c})$, while for patients with low or high serum TBIL level it became $49.4 \%, 28 \%$ and $19.9 \%$ or $23.1 \%, 11.5 \%$ and $11.5 \%$, respectively $(P=0.006$, Fig. $2 d)$.

\section{Discussion}

The presence of PVTT in HCC patients is regarded as indication of an advanced stage, and LR is not recommended [14, 15]. Clinical outcome of patients who require surgical treatment of PVTT predominantly depends on appropriate patient selection, which is complex and involved in several factors. Some patients may not receive liver resection, although they may benefit from it, whereas others receive liver resection but disease still progresses rapidly. Previous studies [11, 12] have reported that physicians often consider locations of PVTT and tumor for HCC patients with PVTT, and several staging methods $[5-7,15]$ have been developed to help predict survival and guide treatment in patients with HCC and PVTT. However, there is still a lack of data for guiding surgeons and cancer patients to select appropriate treatment for HCC patients with PVTT.

In this study, we investigated pre-operative factors associated with short-term or long-term survival in a prospectively observed cohort of 457 patients who underwent surgery for treatment of HCC and PVTT. Our results suggested that patients who died within 3 months after surgery were significantly associated with preoperatively worsened liver function, such as total bilirubin or ascites, compared with those who survived beyond the first 3 months, while patients surviving for $>2$ years after surgery were associated with $\mathrm{CSPH}$ and factors of tumor aggressive nature, such as tumor differentiation, AFP and extent of PVTT.

Our results of analysis for short-term survival patients indicated that patients with poor pre-operative liver 
Table 1 Baseline characteristics for all patients

\begin{tabular}{|c|c|c|c|c|}
\hline Variable & $\begin{array}{l}\begin{array}{l}\text { Overall } \\
(\mathrm{N}=457)\end{array} \\
\end{array}$ & $\begin{array}{l}\text { Alive } \\
(n=139)\end{array}$ & $\begin{array}{l}\text { Died } \\
(n=318)\end{array}$ & $P$ \\
\hline Age, years & $47.9 \pm 10.3$ & $47.6 \pm 9.38$ & $48.1 \pm 10.7$ & 0.612 \\
\hline Sex & & & & 0.378 \\
\hline Female & $50(10.9 \%)$ & $12(8.6 \%)$ & $38(11.9 \%)$ & \\
\hline Male & 407 (89.1\%) & $127(91.4 \%)$ & $280(88.1 \%)$ & \\
\hline \multicolumn{5}{|l|}{ Viral marker } \\
\hline HBsAg positive & $420(92.7 \%)$ & $131(94.2 \%)$ & 289 (92.0\%) & 0.524 \\
\hline Anti-HCV positive & $3(0.7 \%)$ & $1(0.7 \%)$ & $2(0.6 \%)$ & 1.000 \\
\hline AFP, $\mu \mathrm{g} / \mathrm{L}$ & $1210(1.2-777,600)$ & $1067(1.2-37,073)$ & $1210(1.8-777,600)$ & 0.034 \\
\hline AFP, Log $10 \mu \mathrm{g} / \mathrm{L}$ & $3.08(0.08-5.89)$ & $3.03(0.08-4.57)$ & $3.08(0.26-5.89)$ & 0.034 \\
\hline $\mathrm{TBIL}, \mathrm{mg} / \mathrm{dL}$ & $0.81(0.26-7.54)$ & $0.77(0.26-1.87)$ & $0.83(0.30-7.54)$ & 0.023 \\
\hline ALB, g/L & $41.0(17.2-67.2)$ & $41.3(17.2-67.2)$ & $40.8(17.8-65.3)$ & 0.169 \\
\hline PT, s & $12.2(9.6-18.0)$ & $12.2(10.2-15.2)$ & $12.3(9.6-18.0)$ & 0.411 \\
\hline $\mathrm{ALT}, \mathrm{U} / \mathrm{L}$ & $42.6(8.7-187.8)$ & $42.1(11.2-187.8)$ & $42.7(8.7-169.9)$ & 0.965 \\
\hline $\mathrm{PLT}, 10^{9} / \mathrm{L}$ & $156(25.0-629)$ & $156(25.0-369)$ & $156(25.0-629)$ & 0.957 \\
\hline WBC, $10^{9} / \mathrm{L}$ & $5.51(1.44-19.4)$ & $5.40(1.63-11.8)$ & $5.54(1.44-19.4)$ & 0.187 \\
\hline Hemoglobin, g/L & 141 (75.0-195.0) & $142(104.0-174.0)$ & $141(75.0-195.0)$ & 0.988 \\
\hline Child-Pugh class & & & & 0.288 \\
\hline A & 448 (98.0\%) & 138 (99.3\%) & $310(97.5 \%)$ & \\
\hline B & $9(2.0 \%)$ & $1(0.7 \%)$ & $8(2.5 \%)$ & \\
\hline Radiologic ascites & & & & 0.019 \\
\hline No & $422(92.3 \%)$ & 135 (97.1\%) & 287 (90.3\%) & \\
\hline Mild & $35(7.66 \%)$ & $4(2.88 \%)$ & 31 (9.75\%) & \\
\hline Gastroesophageal varices & & & & 1.000 \\
\hline No & $382(83.6 \%)$ & $116(83.5 \%)$ & $266(83.6 \%)$ & \\
\hline Yes & $75(16.4 \%)$ & $23(16.5 \%)$ & $52(16.4 \%)$ & \\
\hline Radiologic spleen length, cm & $11.4(6.30-20.2)$ & $11.0(6.30-17.5)$ & $11.6(6.30-20.2)$ & 0.060 \\
\hline $\mathrm{CSPH}$ & & & & 0.237 \\
\hline No & $233(51.0 \%)$ & $79(56.8 \%)$ & $154(48.4 \%)$ & \\
\hline Mild & $183(40.0 \%)$ & $48(34.5 \%)$ & $135(42.5 \%)$ & \\
\hline Severe & $41(9.0 \%)$ & $12(8.6 \%)$ & $29(9.1 \%)$ & \\
\hline Type of hepatectomy & & & & 0.177 \\
\hline En bloc resection & $289(63.2 \%)$ & $81(58.3 \%)$ & $208(65.4 \%)$ & \\
\hline Non-en bloc resection & $168(36.8 \%)$ & $58(41.7 \%)$ & $110(34.6 \%)$ & \\
\hline Operating time, $\mathrm{h}$ & $2.0(0.45-20.8)$ & $2.0(0.45-20.8)$ & $2.0(0.45-20.8)$ & 0.175 \\
\hline Hilar clamping time, min & $20.0(0-83.0)$ & $21.0(0-48.0)$ & $20.0(0-83.0)$ & 0.248 \\
\hline Intraoperative blood loss, $\mathrm{mL}$ & $400(0-45,000)$ & $400(10-3200)$ & $400(0-45,000)$ & 0.181 \\
\hline Transfusion & & & & 0.229 \\
\hline No & $326(71.3 \%)$ & $105(75.5 \%)$ & $221(69.5 \%)$ & \\
\hline Yes & $131(28.7 \%)$ & $34(24.5 \%)$ & $97(30.5 \%)$ & \\
\hline Surgical margins & & & & 0.115 \\
\hline Ro & $363(79.4 \%)$ & $115(82.7 \%)$ & $248(78.0 \%)$ & \\
\hline $\mathrm{R} 1$ & $16(3.5 \%)$ & $7(5.1 \%)$ & $9(2.8 \%)$ & \\
\hline Unconfirmed & $78(17.1 \%)$ & $17(12.2 \%)$ & $61(19.2 \%)$ & \\
\hline Extent of PVTT & & & & 0.523 \\
\hline Left branch & $102(22.3 \%)$ & $27(19.4 \%)$ & $75(23.6 \%)$ & \\
\hline Right branch & $256(56.0 \%)$ & $83(59.7 \%)$ & $173(54.4 \%)$ & \\
\hline Main trunck/contralateral branch & $99(21.7 \%)$ & $29(20.9 \%)$ & $70(22.0 \%)$ & \\
\hline Tumor number & & & & 0.283 \\
\hline Solitary & $413(90.4 \%)$ & $122(87.8 \%)$ & $291(91.5 \%)$ & \\
\hline
\end{tabular}


Table 1 (continued)

\begin{tabular}{llll}
\hline Variable & $\begin{array}{l}\text { Overall } \\
(\mathbf{N}=\mathbf{4 5 7})\end{array}$ & $\begin{array}{l}\text { Alive } \\
\mathbf{( n = 1 3 9 )}\end{array}$ & $\begin{array}{l}\text { Died } \\
\mathbf{( n = 3 1 8 )}\end{array}$ \\
\hline Multiple & $44(9.6 \%)$ & $17(12.2 \%)$ & $27(8.49 \%)$ \\
$\begin{array}{l}\text { Tumor diameter, cm } \\
\text { Cirrhosis }\end{array}$ & $8.3(0.4-24.0)$ & $7.5(0.6-22.0)$ & $9.0(0.4-24.0)$ \\
No & $131(28.7 \%)$ & & \\
Yes & $326(71.3 \%)$ & $45(32.4 \%)$ & $86(27.0 \%)$ \\
Tumor differentiation & & $94(67.6 \%)$ & $232(73.0 \%)$ \\
II & $24(5.3 \%)$ & $9(6.5 \%)$ & $15(4.7 \%)$ \\
III & $425(93.0 \%)$ & $127(91.4 \%)$ & $298(93.7 \%)$ \\
IV & $8(1.7 \%)$ & $3(2.1 \%)$ & $5(1.6 \%)$ \\
\hline
\end{tabular}

$H B s A g$ hepatitis B surface antigen, anti-HCV hepatitis $C$ virus antibody, AFP a-fetoprotein, TBIL total bilirubin, $A L B$ albumin, $P T$ pro-thrombin time, $A L T$ alanine aminotransferase, PLT platelet count, WBC white blood cell, CSPH clinical significant portal hypertension, PVTT portal vein tumor thrombus

function were not suitable for liver resection, as our results clearly showed worse survival for patients with unfavorable pre-operative parameters, including AFP, TBIL and radiologic ascites. Recovery of liver dysfunction should be recommended first. Radiologic ascites become a predominant prognostic variable for decision making. In clinical situation, although for patients with Child A class, ascites is common. This implied we can more accurately select candidates for liver resection, even for patients with Child A class. There is a research reporting that CTP class can affect the survival of HCC patients with PVTT [16], which is similar to our findings.

Longer-term survivors often did not have hepatic decompensation, while the patients who had CSPH always exhibited worse long-term outcome. There is a similar research reporting that the risk of 3-year and 5 -year mortality can be increased by the presence of $\mathrm{CSPH}$, which also increases the risk of postoperative clinical decompensation [17]. The factors also indicated that tumor aggressive nature, such as tumor differentiation, AFP and extent of PVTT, were tightly associated with long-term survival of patients. Of the four factors, CSPH, AFP and extent of PVTT can be obtained preoperatively, and according to this we can make recommendation that resection treatment was not suitable for patients with high AFP, PVTT in the main portal vein, and CSPH. The factor tumor differentiation is a pathological indicator and can only be obtained after surgery, which may provide reference for whether adjuvant therapy after surgery should be given or not.

Our result showed that serum AFP level was a key factor of both short-term and long-term survivals for patients with PVTT. We further investigated the staining pattern of AFP in both HCC tissues and peritumoral tissues according to immunohistochemical analysis (Additional file 2: Supplementary method). The immunostaining results showed the expression of AFP in short-term survivors was higher than that in long-term survivors, which were consistent with the serum AFP results of this study (Additional file 3: Figure S1). Furthermore, the expression of AFP was elevated in tumor tissues, while it was decreased in peritumoral tissues, which was similar to previous study [18].

Liver cirrhosis is common in patients with advanced HCC [19], and a large proportion of cirrhosis was also observed in patients with PVTT of this study. To evaluate whether the cirrhosis context was associated with the effect of risk factors on survival in long-term survivors, we conducted survival analysis for AFP and TBIL level in subset of cirrhosis. Survival analysis showed that AFP is a risk factor for prognosis in both patients with and without cirrhosis. However, TBIL was a risk factor only in patients without cirrhosis, and the effect of TBIL on prognosis was diminished in the context of cirrhosis. This results suggested that although cirrhosis was not a risk factor for prognosis in patients with PVTT, the existence of cirrhosis made some liver function factors, such as TBIL, no longer a risk factor for prognosis of long-term survivors. However, the presence of cirrhosis did not affect the characteristics of the tumor itself, such as AFP.

Although our current study showed consistency over multiple pre-operative predictors of improved survival for patients, several biases might be present. First, although each participating center provided up-to-date information on vital statistics, inaccuracies in the actual status of patients could not be ruled out as many patients were referred back to their own referring hospitals. As the chance of a dead patient had higher possibility of being lost during follow-up in the database than the opposite, this potential inaccuracy could lead to an overestimation of effect of the end points. Second, the majority of patients had a background of HBV infection, which might 
Table 2 Descriptive analysis of survivors versus non-survivors at 3 months

\begin{tabular}{|c|c|c|c|}
\hline Variable & $\begin{array}{l}\text { Alive } \\
(n=405)\end{array}$ & $\begin{array}{l}\text { Died } \\
(n=51)\end{array}$ & $P$ \\
\hline Age, years & $47.8 \pm 10.2$ & $48.9 \pm 10.6$ & 0.453 \\
\hline Sex & & & 1.000 \\
\hline Female & 44 (10.9\%) & $6(11.8 \%)$ & \\
\hline Male & 361 (89.1\%) & 45 (88.2\%) & \\
\hline \multicolumn{4}{|l|}{ Viral marker } \\
\hline HBsAg positive & $370(92.0 \%)$ & 49 (98.0\%) & 0.156 \\
\hline Anti-HCV positive & $3(0.7 \%)$ & $0(0.0 \%)$ & 1.000 \\
\hline $\mathrm{AFP}, \mu \mathrm{g} / \mathrm{L}$ & $1210(1.2-276,354)$ & $1210(2.9-777,600)$ & 0.003 \\
\hline AFP, Log $10 \mu \mathrm{g} / \mathrm{L}$ & $3.08(0.08-5.44)$ & $3.08(0.46-5.89)$ & 0.003 \\
\hline $\mathrm{TBIL}, \mathrm{mg} / \mathrm{dL}$ & $0.79(0.26-7.54)$ & $0.94(0.34-7.54)$ & $<0.001$ \\
\hline$A L B, g / L$ & $40.9(17.2-67.2)$ & $41.6(32.4-65.3)$ & 0.799 \\
\hline PT, s & $12.2(9.6-18.0)$ & $12.4(10.7-18.0)$ & 0.121 \\
\hline$A L T, U / L$ & $43.3(8.7-187.8)$ & $41.3(9.3-162.1)$ & 0.853 \\
\hline $\mathrm{PLT}, 10^{9} / \mathrm{L}$ & $157(25.0-629)$ & $139(63.0-358)$ & 0.452 \\
\hline $\mathrm{WBC}, 10^{9} / \mathrm{L}$ & $5.51(1.44-19.4)$ & $5.41(2.29-10.6)$ & 0.964 \\
\hline Hemoglobin, g/L & $141(94.0-195.0)$ & $146(75.0-172.0)$ & 0.085 \\
\hline Child-Pugh class & & & 0.266 \\
\hline A & 398 (98.3\%) & 49 (96.1\%) & \\
\hline B & $7(1.7 \%)$ & $2(3.9 \%)$ & \\
\hline Radiologic ascites & & & 0.003 \\
\hline No & $380(93.8 \%)$ & 41 (80.4\%) & \\
\hline Mild & $25(6.2 \%)$ & $10(19.6 \%)$ & \\
\hline Gastroesophageal varices & & & 0.397 \\
\hline No & 341 (84.2\%) & $40(78.4 \%)$ & \\
\hline Yes & $64(15.8 \%)$ & $11(21.6 \%)$ & \\
\hline Radiologic spleen length, cm & $11.3(6.3-20.2)$ & $12.0(8.0-18.9)$ & 0.029 \\
\hline $\mathrm{CSPH}$ & & & 0.194 \\
\hline No & $212(52.3 \%)$ & $20(39.2 \%)$ & \\
\hline Mild & $157(38.8 \%)$ & $26(51.0 \%)$ & \\
\hline Severe & $36(8.9 \%)$ & $5(9.8 \%)$ & \\
\hline Type of hepatectomy & & & 0.327 \\
\hline Enbloc & $253(62.5 \%)$ & $36(70.6 \%)$ & \\
\hline Non-enbloc & $152(37.5 \%)$ & 15 (29.4\%) & \\
\hline Operating time, $\mathrm{h}$ & $2.0(0.45-20.8)$ & $2.0(0.45-5.7)$ & 0.862 \\
\hline Hilar clamping time, min & $20.0(0-83.0)$ & $20.0(0-51.0)$ & 0.474 \\
\hline Intraoperative blood loss, mL & $400(0-45,000)$ & $400(0-8000)$ & 0.598 \\
\hline Transfusion & & & 0.544 \\
\hline No & 291 (71.9\%) & $34(66.7 \%)$ & \\
\hline Yes & $114(28.1 \%)$ & $17(33.3 \%)$ & \\
\hline Surgical margins & & & 1.000 \\
\hline RO & $321(79.3 \%)$ & $42(82.3 \%)$ & \\
\hline R1 & $15(3.7 \%)$ & $1(2.0 \%)$ & \\
\hline Unconfirmed & $69(17.0 \%)$ & $8(15.7 \%)$ & \\
\hline Extent of PVTT & & & 0.281 \\
\hline Left branch & $95(23.5 \%)$ & $7(13.7 \%)$ & \\
\hline Right branch & $224(55.3 \%)$ & $31(60.8 \%)$ & \\
\hline Main trunck/contralateral branch & $86(21.2 \%)$ & $13(25.5 \%)$ & \\
\hline Tumor number & & & 1.000 \\
\hline Solitary & $366(90.4 \%)$ & $46(90.2 \%)$ & \\
\hline
\end{tabular}


Table 2 (continued)

\begin{tabular}{llll}
\hline Variable & $\begin{array}{l}\text { Alive } \\
(\mathbf{n = 4 0 5 )}\end{array}$ & $\begin{array}{l}\text { Died } \\
(\mathbf{n}=\mathbf{5 1})\end{array}$ \\
\hline Multiple & $39(9.6 \%)$ & $5(9.8 \%)$ & $\mathbf{P}$ \\
Tumor diameter, cm & $8.0(0.6-22.0)$ & $10.0(0.4-24.0)$ & \\
Cirrhosis & & $10(19.6 \%)$ \\
$\quad$ No & $121(29.9 \%)$ & $41(80.4 \%)$ \\
Yes & $284(70.1 \%)$ & \\
Tumor differentiation & & $1(2.0 \%)$ \\
II & $23(5.7 \%)$ & $49(96.0 \%)$ \\
III & $375(92.6 \%)$ & $1(2.0 \%)$ \\
IV & $7(1.7 \%)$ & 0.612 \\
\hline
\end{tabular}

HBsAg hepatitis B surface antigen, anti-HCV hepatitis C virus antibody, AFP a-fetoprotein, TBIL total bilirubin, $A L B$ albumin, $P T$ pro-thrombin time, ALT alanine aminotransferase, PLT platelet count, WBC white blood cell, CSPH clinical significant portal hypertension, $P V T T$ portal vein tumor thrombus

be not suitable for patients with other background. The common factor for all patients was the need of surgical
Methods

Patients

Table 3 Multivariable logistic regression analysis exploring factors associated with death within 3 or 24 months after hepatectomy

\begin{tabular}{|c|c|c|c|c|}
\hline \multirow[t]{2}{*}{ Variable } & \multicolumn{2}{|l|}{ Three month analysis } & \multicolumn{2}{|l|}{ Two year analysis } \\
\hline & Odds ratio $(95 \% \mathrm{Cl})$ & $P$ & Odds ratio $(95 \% \mathrm{CI})$ & $P$ \\
\hline AFP, Log $10 \mu \mathrm{g} / \mathrm{L}$ & $1.60(1.15$ to 2.25$)$ & 0.010 & $1.34(1.06$ to 1.69$)$ & 0.034 \\
\hline $\mathrm{TBIL}, \mathrm{mg} / \mathrm{dL}$ & 1.94 (1.21 to 3.12$)$ & 0.026 & & \\
\hline Radiologic ascites, positive vs. negative & $3.72(1.59$ to 8.15$)$ & 0.009 & & \\
\hline CSPH, yes vs. no & & & $1.75(1.10$ to 2.81$)$ & 0.016 \\
\hline Extent of PVTT: main trunck vs. left/right branch & & & 1.81 (0.99 to 3.53) & 0.043 \\
\hline Tumor differentiation: III-IV vs. II & & & $3.93(1.56$ to 10.18$)$ & 0.017 \\
\hline
\end{tabular}

AFP a-fetoprotein, TBIL total bilirubin, CSPH clinical significant portal hypertension, PVTT portal vein tumor thrombus

treatment. For each patient, indication to proceed with surgery was based on the need to alleviate the symptoms of portal hypertension and a presumed life expectancy of $>3$ months. All these factors could be biased by the experience and preference of the anesthesiologist, surgeon, oncologist, institutional preference and patient wishes.

In conclusion, this large prospective cohort study strongly suggested that survival depended on general preoperative status for liver resection of HCC and PVTT. In particular, serum AFP level, TBIL level, and radiologic ascites were significantly and independently associated with poor short-term survival ( $<3$ months), while serum AFP level, tumor differentiation, CSPH, and extent of PVTT were significantly and independently associated with longer-term survival.
A total of 457 consecutive HCC patients with PVTT who underwent LR at the Eastern Hepatobiliary Surgery Hospital (EHBH) from January 2008 to December 2010 were enrolled in the present study, and their electronic medical records were systematically reviewed. All patients were histologically diagnosed as HCC. Our retrospective analysis of data was approved by the Institutional Ethics Committee of the EHBH. Informed consent was obtained from all the patients before surgery.

All surgical team members adhered to the principle of performing LR for individuals whose life expectancy should be no less than 3 months.

\section{LR procedure}

Based on the location and extent of PVTT, thrombectomy was carried out. The en bloc resection was considered if there was a sufficient safety margin between its root and the tip of the thrombus. Once the PVTT protruded into the main portal vein beyond the resection 
Table 4 Descriptive analysis of survivors versus non-survivors at 2 years

\begin{tabular}{|c|c|c|c|}
\hline Variable & $\begin{array}{l}\text { Alive } \\
(n=98)\end{array}$ & $\begin{array}{l}\text { Died } \\
(n=291)\end{array}$ & $P$ \\
\hline Age, years & $48.9 \pm 11.1$ & $47.7 \pm 10.4$ & 0.368 \\
\hline Sex & & & 1.000 \\
\hline Female & $11(11.2 \%)$ & $34(11.7 \%)$ & \\
\hline Male & 87 (88.8\%) & $257(88.3 \%)$ & \\
\hline \multicolumn{4}{|l|}{ Viral marker } \\
\hline HBsAg positive & $87(89.7 \%)$ & $266(92.4 \%)$ & 0.541 \\
\hline Anti-HCV positive & $0(0.0 \%)$ & $2(0.7 \%)$ & 1.000 \\
\hline $\mathrm{AFP}, \mu \mathrm{g} / \mathrm{L}$ & $763(1.2-138,410)$ & $1210(1.8-777,600)$ & 0.012 \\
\hline AFP, Log $10 \mu \mathrm{g} / \mathrm{L}$ & $2.88(0.08-5.14)$ & $3.08(0.26-5.89)$ & 0.012 \\
\hline $\mathrm{TBIL}, \mathrm{mg} / \mathrm{dL}$ & $0.77(0.26-1.87)$ & $0.85(0.30-7.54)$ & 0.024 \\
\hline$A L B, g / L$ & $41.6(17.2-67.2)$ & $40.8(17.8-65.3)$ & 0.421 \\
\hline PT, s & $12.1(9.9-14.9)$ & $12.4(9.6-18.0)$ & 0.054 \\
\hline$A L T, U / L$ & $42.8(11.2-187.8)$ & $43.0(8.7-169.9)$ & 0.875 \\
\hline $\mathrm{PLT}, 10^{9} / \mathrm{L}$ & $172(25.0-405)$ & $151(25.0-629)$ & 0.148 \\
\hline $\mathrm{WBC}, 10^{9} / \mathrm{L}$ & $5.57(1.80-10.4)$ & $5.44(1.44-19.4)$ & 0.672 \\
\hline Hemoglobin, g/L & $140(104-174)$ & $141(75.0-195)$ & 0.984 \\
\hline Child-Pugh class & & & 0.460 \\
\hline A & 97 (99.0\%) & $283(97.3 \%)$ & \\
\hline B & $1(1.0 \%)$ & $8(2.7 \%)$ & \\
\hline Radiologic ascites & & & 0.110 \\
\hline No & 94 (95.9\%) & $262(90.0 \%)$ & \\
\hline Mild & $4(4.1 \%)$ & $29(10.0 \%)$ & \\
\hline Gastroesophageal varices & & & 0.155 \\
\hline No & 88 (89.8\%) & $242(83.2 \%)$ & \\
\hline Yes & $10(10.2 \%)$ & $49(16.8 \%)$ & \\
\hline Radiologic spleen length, cm & $11.0(6.3-16.4)$ & $11.7(6.3-20.2)$ & 0.005 \\
\hline $\mathrm{CSPH}$ & & & 0.010 \\
\hline No & $60(61.2 \%)$ & $138(47.4 \%)$ & \\
\hline Mild & $36(36.7 \%)$ & $124(42.6 \%)$ & \\
\hline Severe & $2(2.1 \%)$ & $29(10.0 \%)$ & \\
\hline Type of hepatectomy & & & 0.130 \\
\hline En bloc resection & $56(57.1 \%)$ & $193(66.3 \%)$ & \\
\hline Non-en bloc resection & $42(42.9 \%)$ & $98(33.7 \%)$ & \\
\hline Operating time, $\mathrm{h}$ & $2.0(0.45-20.8)$ & $2.0(0.45-10.3)$ & 0.488 \\
\hline Hilar clamping time, min & $21.0(0-58.0)$ & $20.0(0-83.0)$ & 0.334 \\
\hline Intraoperative blood loss, mL & $325(100-3200)$ & $400(0-45,000)$ & 0.038 \\
\hline Transfusion & & & 0.409 \\
\hline No & $73(74.5 \%)$ & $202(69.4 \%)$ & \\
\hline Yes & $25(25.5 \%)$ & $89(30.6 \%)$ & \\
\hline Surgical margins & & & 0.690 \\
\hline RO & $81(82.7 \%)$ & $227(78.0 \%)$ & \\
\hline R1 & $2(2.0 \%)$ & $9(3.1 \%)$ & \\
\hline Unconfirmed & $15(15.3 \%)$ & $55(18.9 \%)$ & \\
\hline Extent of PVTT & & & 0.115 \\
\hline Left branch & $22(22.4 \%)$ & $69(23.7 \%)$ & \\
\hline Right branch & $62(63.3 \%)$ & $154(52.9 \%)$ & \\
\hline Main trunck/contralateral branch & $14(14.3 \%)$ & $68(23.4 \%)$ & \\
\hline Tumor number & & & 0.781 \\
\hline Solitary & 88 (89.8\%) & $266(91.4 \%)$ & \\
\hline
\end{tabular}


Table 4 (continued)

\begin{tabular}{llll}
\hline Variable & $\begin{array}{l}\text { Alive } \\
(\mathbf{n = 9 8 )}\end{array}$ & $\begin{array}{l}\text { Died } \\
(\mathbf{n = 2 9 1 )}\end{array}$ \\
\hline Multiple & $10(10.2 \%)$ & $25(8.59 \%)$ \\
Tumor diameter, cm & $7.7(2.0-18.2)$ & $8.8(0.4-24.0)$ & \\
Cirrhosis & & $74(25.4 \%)$ \\
$\quad$ No & $35(35.7 \%)$ & $217(74.6 \%)$ \\
Yes & $63(64.3 \%)$ & \\
Tumor differentiation & & $9(3.09 \%)$ \\
II & $11(11.2 \%)$ & $277(95.2 \%)$ \\
III & $85(86.7 \%)$ & $5(1.72 \%)$ \\
IV & $2(2.1 \%)$ & 0.0679 \\
\hline
\end{tabular}

HBsAg hepatitis B surface antigen, anti-HCV hepatitis C virus antibody, AFP a-fetoprotein, TBIL total bilirubin, $A L B$ albumin, $P T$ pro-thrombin time, ALT alanine aminotransferase, PLT platelet count, WBC white blood cell, CSPH clinical significant portal hypertension, $P V T T$ portal vein tumor thrombus

line, PVTT was removed from the opened stump of the portal vein. The main portal trunk was exposed and clamped from distal to the PVTT if the PVTT extended into the main portal trunk and its primary branches on both sides. An incision was made at the bifurcation of the right and left portal veins to extract the PVTT. The incision was flushed with normal saline, and the presence of PVTT was confirmed. Subsequently, the stump was closed by a continuous suture.

The commonly used methods of surgical resection are as follows. (1) Segmental hepatectomy: HCC is confined to the segment of liver, and PVTT in the segmental branches of portal vein can be excised in the same segment, which is suitable for type I and partial II PVTT patients. (2) Hemi-hepatectomy: HCC is located on the left or right lobe of liver, and PVTT in the first branch of portal vein can be excised in the same half-liver, which is suitable for partial patients with type II and III PVTT. (3) Hepatectomy plus thrombectomy: PVTT extends to the bifurcation of the right and left portal veins, or to the main portal vein, which is suitable for patients with type III PVTT. In the hepatectomy for HCC, concomitant thrombectomy is performed with temporary occlusion of the portal veins before the tumor thrombus is removed from the vessels. An incision of portal vein is carried out at the bifurcation of the right and left portal veins, and then PVTT is extracted from the incision in the portal vein. If there is a sufficient safety margin between its root and the tip of the thrombus for portal vein ligation, the en bloc resection, including the bifurcation with or without the main and/or contralateral portal veins, can be used for HCC patients with PVTT. (4) Portal vein resection and reconstruction: PVTT invades the main portal vein wall and is difficult to be removed, which is suitable for some patients with type III and IV PVTT. In the hepatectomy, the invaded main portal vein may be removed together with PVTT, and the 6/0 Prolene continuous suture with a $1-\mathrm{cm}$ growth factor was used in direct end-to-end portal vein anastomosis. If the resected portal vein is too long, the autologous vein or artificial blood vessel can be used as graft. Hepatic artery and portal vein chemotherapy pump implantation are suitable for the patients with PVTT removed in the hepatectomy, and the pathway for the postoperative regional chemotherapy is reserved by hepatic artery and portal vein chemotherapy pump during the operation. Direct suction or back-bleeding is conducted if there is any residual tumor thrombus in the branch of portal vein. Residual tissue containing potential tumor thrombus should be removed by flushing the portal vein lumen with water and heparinized saline.

\section{Definitions of resection type}

R0 resection was defined by the absence of microscopic tumor invasion of the resection margin; R1 resection was defined as a complete macroscopic resection with a positive pathological margin; and R2 resection was defined as a macroscopically positive margin.

\section{Classification of PVTT}

The criteria used by Chen et al. [9] were employed in the present study, and the types of PVTT were classified into three subgroups as follows: (a) type A was defined as PVTT in the main portal vein; (b) type B was defined as PVTT in the first-order portal vein branch (the right or left portal vein); and (c) type $\mathrm{C}$ was defined as PVTT in the second- or lower-order portal vein branch (segmental branches of portal vein or higher). 

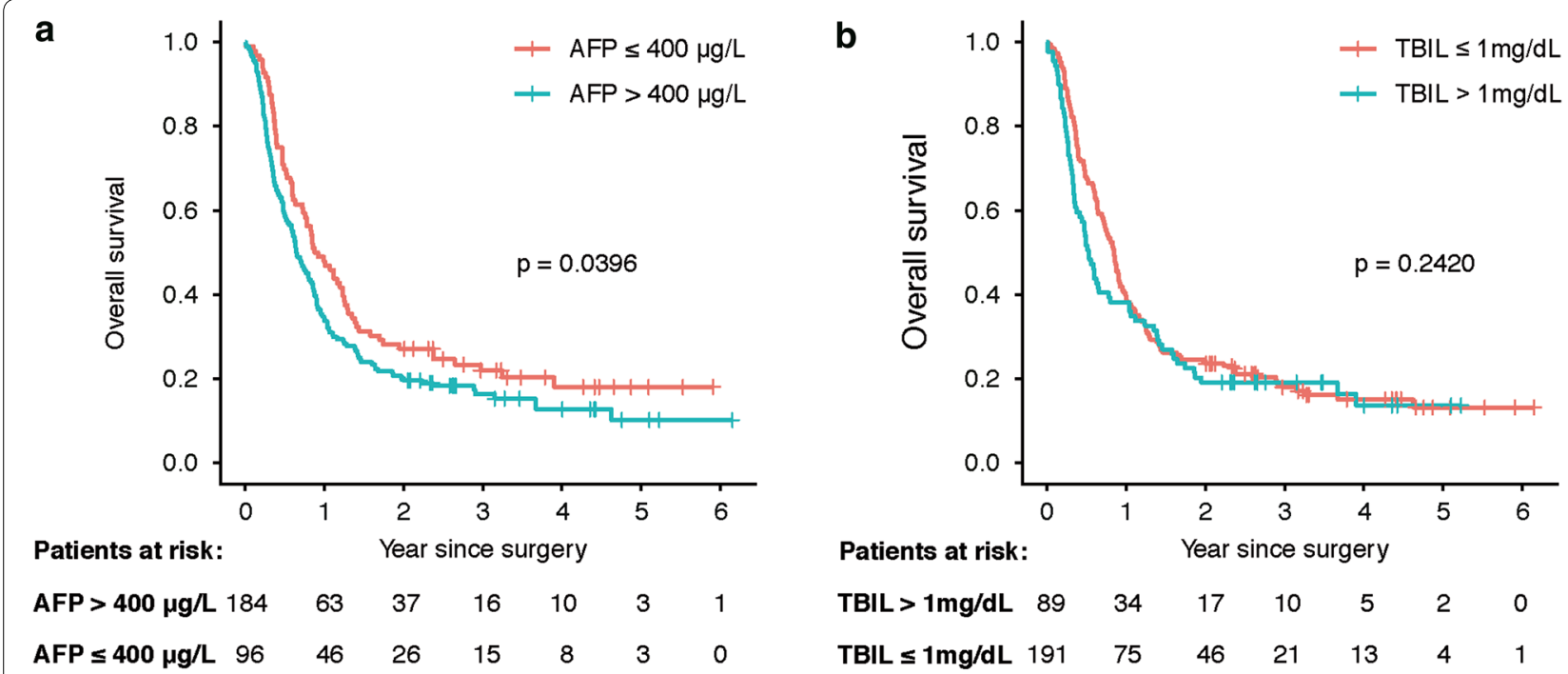

C

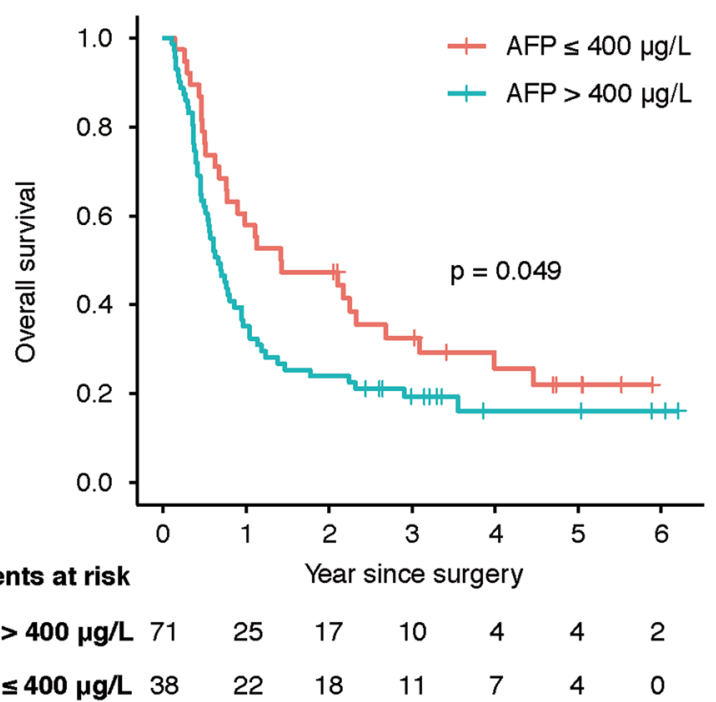

d

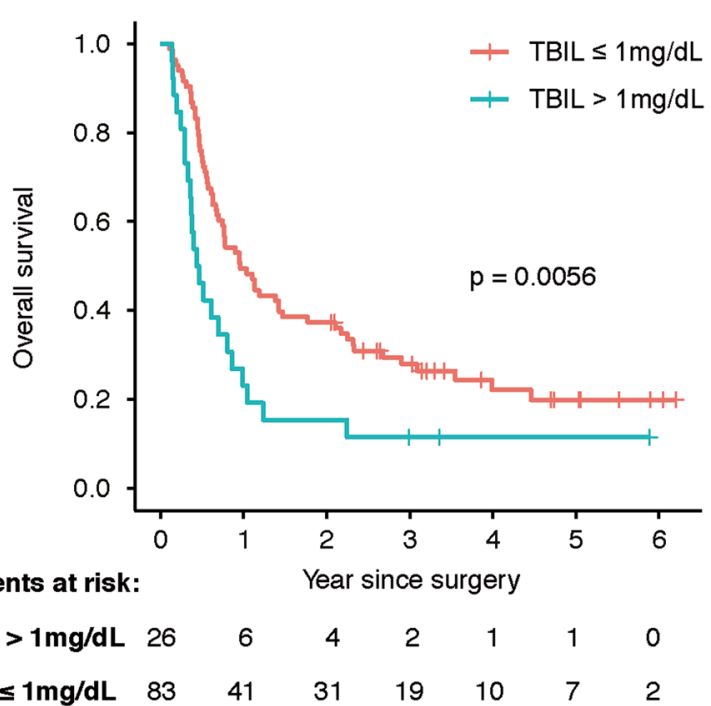

Fig. 2 Survival curve of OS for cirrhosis subgroup. a Survival curve for AFP level in patients with cirrhosis. b Survival curve for TBIL level in patients with cirrhosis. c Survival curve for AFP level in patients without cirrhosis. d Survival curve for TBIL level in patients without cirrhosis. OS overall survival

\section{Follow-up and endpoints}

Survival at 3 months post-operation was the primary outcome, and survival at 2 years post-operation was the secondary outcome. To ensure reliability of primary and secondary outcomes, all the case records were reviewed by participating investigators after closure of the database with special emphasis on vital statistics, no matter dead or alive for each patient. OS was also calculated from the date of surgery to the date of patient death or last follow-up.

\section{Statistical analysis}

Statistical analysis was performed using the $\mathrm{R}$ statistical package v.3.2.1 (http://www.r-project.org/). Continuous data were expressed as mean $\pm \mathrm{SD}$ or median (range), and comparisons were conducted using the Student's t-test or Mann-Whitney U test. The Pearson's $\chi^{2}$ test or Fisher's exact test was used to compare categorical data. Data distributions before analysis of results and calculations of statistical parameters were reviewed. Factors associated with death within 3 months were assessed by univariable and multivariable logistic regression analyses of cases without missing data since hepatectomy and 
factors associated with survival after surgery for $>2$ years. Survival curves were established using the Kaplan-Meier method and compared using the log-rank test. P values $<0.05$ were considered statistically significant.

\section{Additional files}

Additional file 1: Table S1. Univariable logistic regression analysis exploring factors associated with death within 3 or 24 months after hepatectomy.

Additional file 2. Supplementary method.

Additional file 3: Figure S1. AFP expression in HCC tissue, paired peritumoral tissue, and normal liver tissue. (A) AFP expression in control liver tissue, adjacent tissue $<1 \mathrm{~cm}$ from tumor, tissue at operative site $(>1$ $\mathrm{cm}$ from tumor or more), and tumor tissue for long-term and short-term survivors. Tissue specimens were immunostained with antibody directed against AFP (GB11287, Servicebio, Shanghai, China). Representative examples are shown. Panel B-C show AFP expression in control livers of non-HCC patients. Panels D-F show AFP expression in long term HCC patient of adjacent tissue $<1 \mathrm{~cm}$ from tumor (D), tissue at operative site (E), and tumor tissue (F). Panels G-I show AFP expression in short term HCC patient of adjacent tissue $<1 \mathrm{~cm}$ from tumor $(\mathrm{G})$, tissue at operative site $(\mathrm{H})$, and tumor tissue (I).

\section{Abbreviations}

PVTT: portal vein tumor thrombosis; HCC: hepatocellular carcinoma; LR: liver resection; OS: overall survival; AFP: alpha-fetoprotein; TBIL: total bilirubin; OR: odds ratio; CSPH: clinically significant portal hypertension; CTP: Child-Turcotte-Pugh; ECOG: electrocorticography; SD: standard deviation.

\section{Authors' contributions}

$\mathrm{NJ}$ and $\mathrm{YX}$ contributed for study concept and design. ZY, YX, RG and YW performed statistical analysis. LH, WW and ZL drafted the article. WW, ZL, LH, $\mathrm{NJ}$ and $\mathrm{YX}$ critically revised the article. All authors read and approved the final manuscript.

\section{Author details \\ ${ }^{1}$ Department of Hepatic Surgery IV, The Eastern Hepatobiliary Surgery Hospital, Second Military Medical University, 225 Changhai Road, Shang- hai 200433, China. ${ }^{2}$ Department of Radiotherapy, The Eastern Hepatobiliary Surgery Hospital, Second Military Medical University, Shanghai 200438, China. ${ }^{3}$ Department of General Surgery, The Zhongda Hospital, Southeast University, Nanjing 210009, China.}

\section{Acknowledgements}

Not applicable.

\section{Competing interests}

The authors reported no proprietary or commercial interest in any product mentioned or concept discussed in this article.

\section{Availability of data and materials}

The datasets generated during and/or analyzed during the current study are available from the corresponding author on reasonable request.

\section{Consent for publication}

Not applicable.

\section{Ethics approval and consent to participate}

Our retrospective analysis of data was approved by the Institutional Ethics Committee of the EHBH. Informed consent was obtained from all the patients before surgery.

\section{Funding}

This study was supported by the Natural Science and Medical Guidance Foundation of Shanghai (16ZR1400100, 16411966200), and the Foundation of Shanghai Health and Family Planning Commission (201540381, 2018BR34).

\section{Publisher's Note}

Springer Nature remains neutral with regard to jurisdictional claims in published maps and institutional affiliations.

Received: 19 October 2018 Accepted: 26 February 2019

Published online: 06 March 2019

\section{References}

1. Thomas MB, Jaffe D, Choti MM, Belghiti J, Curley S, Fong Y, et al. Hepatocellular carcinoma: consensus recommendations of the national cancer institute clinical trials planning meeting. J Clin Oncol. 2010;28:3994-4005.

2. Pirisi M, Avellini C, Fabris C, Scott C, Bardus P, Soardo G, et al. Portal vein thrombosis in hepatocellular carcinoma: age and sex distribution in an autopsy study. J Cancer Res Clin Oncol. 1998;124:397-400.

3. Llovet JM, Bustamante J, Castells A, Vilana R, Ayuso MC, Sala M, et al. Natural history of untreated nonsurgical hepatocellular carcinoma: rationale for the design and evaluation of therapeutic trials. Hepatology. 1999;29:62-7.

4. Cheng A-L, Kang Y-K, Chen Z, Tsao C-J, Qin S, Kim JS, et al. Efficacy and safety of sorafenib in patients in the Asia-Pacific region with advanced hepatocellular carcinoma: a phase III randomised, double-blind, placebocontrolled trial. Lancet Oncol. 2009:10:25-34.

5. Omata M, Lesmana LA, Tateishi R, Chen P-J, Lin S-M, Yoshida H, et al. Asian Pacific Association for the Study of the Liver consensus recommendations on hepatocellular carcinoma. Hepatol Int. 2010;4:439-74.

6. Yau T, Tang VYF, Yao T-J, Fan S-T, Lo C-M, Poon RTP. Development of Hong Kong Liver Cancer staging system with treatment stratification for patients with hepatocellular carcinoma. Gastroenterology. 2014;146:1691-1700.e1693.

7. Kokudo N, Hasegawa K, Akahane M, Igaki H, Izumi N, Ichida T, et al. Evidence-based clinical practice guidelines for hepatocellular carcinoma. Hepatol Res. 2015;45:123-7.

8. Inoue Y, Hasegawa K, Ishizawa T, Aoki T, Sano K, Beck Y, et al. Is there any difference in survival according to the portal tumor thrombectomy method in patients with hepatocellular carcinoma? Surgery. 2009;145:9-19.

9. Chen X-P, Qiu F-Z, Wu Z-D, Zhang Z-W, Huang Z-Y, Chen Y-F, et al. Effects of location and extension of portal vein tumor thrombus on long-term outcomes of surgical treatment for hepatocellular carcinoma. Ann Surg Oncol. 2006;13:940-6.

10. Liu P-H, Lee $Y-H$, Hsia C-Y, Hsu C-Y, Huang Y-H, Chiou Y-Y, et al. Surgical resection versus transarterial chemoembolization for hepatocellular carcinoma with portal vein tumor thrombosis: a propensity score analysis. Ann Surg Oncol. 2014;21:1825-33.

11. Ye J-Z, Zhang Y-Q, Ye H-H, Bai T, Ma L, Xiang B-D, et al. Appropriate treatment strategies improve survival of hepatocellular carcinoma patients with portal vein tumor thrombus. World J Gastroenterol. 2014;20:17141-7.

12. Shi J, Lai EH, Li N, Guo W-X, Xue J, Lau WY, et al. Surgical treatment of hepatocellular carcinoma with portal vein tumor thrombus. Ann Surg Oncol. 2010;17:2073-80.

13. Ye J-Z, Wang Y-Y, Bai T, Chen J, Xiang B-D, Wu F-X, et al. Surgical resection for hepatocellular carcinoma with portal vein tumor thrombus in the Asia-Pacific region beyond the Barcelona Clinic Liver Cancer treatment algorithms: a review and update. Oncotarget. 2017;8:93258-78.

14. Forner A, Reig M, Bruix J. Hepatocellular carcinoma. Lancet. 2018:391:1301-14.

15. Bruix J, Gores GJ, Mazzaferro V. Hepatocellular carcinoma: clinical frontiers and perspectives. Gut. 2014;63:844-55.

16. Lee JM, Jang BK, Lee YJ, Choi WY, Choi SM, Chung WJ, et al. Survival outcomes of hepatic resection compared with transarterial chemoembolization or sorafenib for hepatocellular carcinoma with portal vein tumor thrombosis. Clin Mol Hepatol. 2016;22:160-7. 
17. Berzigotti A, Reig M, Abraldes JG, Bosch J, Bruix J. Portal hypertension and the outcome of surgery for hepatocellular carcinoma in compensated cirrhosis: a systematic review and meta-analysis. Hepatology. 2015;61:526-36.

18. Mitsuhashi N, Kobayashi S, Doki T, Kimura F, Shimizu H, Yoshidome $\mathrm{H}$, et al. Clinical significance of alpha-fetoprotein: involvement in proliferation, angiogenesis, and apoptosis of hepatocellular carcinoma. J Gastroenterol Hepatol. 2008;23:189-97.

19. Bruix J, Sherman MD. American Association for the study of liver, management of hepatocellular carcinoma: an update. Hepatology. 2011;53:1020-2.
Ready to submit your research? Choose BMC and benefit from:

- fast, convenient online submission

- thorough peer review by experienced researchers in your field

- rapid publication on acceptance

- support for research data, including large and complex data types

- gold Open Access which fosters wider collaboration and increased citations

- maximum visibility for your research: over 100M website views per year

At BMC, research is always in progress.

Learn more biomedcentral.com/submissions 